\title{
Anesthesiological considerations in emphysema surgery
}

\author{
Philip Woldt, Philipp Kruse, Bjoern Ellger \\ Department for Anesthesiology, Intensive Care Medicine and Pain Therapy, Klinikum Westfalen, Dortmund, Germany \\ Contributions: (I) Conception and design: All authors; (II) Administrative support: B Ellger; (III) Provision of study material or patients: None; (IV) \\ Collection and assembly of data: None; (V) Data analysis and interpretation: None; (VI) Manuscript writing: All authors; (VII) Final approval of \\ manuscript: All authors. \\ Correspondence to: Prof. Dr. Bjoern Ellger, MD, PhD. Department for Anesthesiology, Intensive Care Medicine and Pain Therapy, Klinikum \\ Westfalen, Am Knappschaftskrankenhaus 1, D-44309 Dortmund, Germany. Email: bjoern.ellger@klinikum-westfalen.de.
}

\begin{abstract}
In the last decades, developing thoracic surgery raised the demands for sophisticated anesthesiological management. Especially patients with end-stage thoracic emphysema challenge the anesthesiologist to make modern surgery possible and to provide a safe and effective perioperative management. The development and scientific work-up of single lung ventilation (SLV) laid the cornerstone for surgery of the non-ventilated lung and hemi-thorax. However, modern medicine extended surgical options to extensive tracheal surgery and to patients suffering from severely insufficient lung-capacity precluding single-lung ventilation or artificial ventilation in se. For those critically ill, different techniques were thus developed and evaluated in recent research, among others, non-intubated surgery and surgery under extracorporeal perfusion support that temporarily avoids pulmonary gas exchange and ventilation via the trachea in any way. To tackle postoperative pain with its successive problems of immobilization, insufficient respiration and airway-clearance, regional anesthesia offers great advantages. Thoracic epidural anesthesia (TEA) is considered as the gold standard; complementary, modern ultrasound techniques make regional anesthesia possible even when contraindications prohibit neuraxial blocks. Especially paravertebral block, musculus serratus anterior block, intercostal block and the musculus erector spinae block provide good postoperative pain relief and appear to influence chronic post-thoracotmy pain positively. Careful preoperative preparation, intraoperative monitoring and patient-tailored, individual perioperative management by a well-trained team ensure good results, a good survival and favorable quality of life. This article provides a brief overview over state-of-the-art techniques and future perspectives to provide anesthesia in emphysema surgery.
\end{abstract}

Keywords: Thoracic anesthesia; non-intubated surgery; extracorporeal circulation; regional anesthesia

Submitted Apr 16, 2020. Accepted for publication Jul 22, 2020.

doi: 10.21037/atm-2019-le-06

View this article at: http://dx.doi.org/10.21037/atm-2019-le-06

\section{Introduction}

Thoracic surgery became possible after general anesthesia, ventilation in negative-pressure chambers and positive pressure ventilation became more sophisticated in the early years of the $20^{\text {th }}$ century. The breakthrough came stepwise with the development of endobronchial blockers and special double-lumen endobronchial tubes to allow single lung ventilation (SLV) in the $1950^{\text {th }}$, these devices placed under general anesthesia assured advantageous conditions for surgery, a block of coughing, oxygenation and decarboxylation and a reasonable patient comfort (1). However, injuries of the airways and lungs (baro-, volu-, atelect-, bio- and pressure-trauma) and an impairment of cardial performance are inevitable connected to the procedures. Thus thoracic anesthesia belongs into expert hands to avoid complications turning into catastrophes: Especially unexpected difficult airway situations, hypoxia, airway injury, hemorrhage, arrhythmia and cardiac arrest as well as re-expansion edema and (tension)pneumothorax 
are reported as treatable complications closely linked to the surgical procedure (2). Postoperatively, impaired neuromuscular performance, impaired neurocognitive function, (persistent) pain and a high risk for pneumonia expose the patient to a significant morbidity and mortality.

As a matter of fact, advancing anesthesiological techniques, some of which we will present in this review, improve comfort and safety. The advancements open doors to surgical options even in patients with severely impaired pulmonary function. Not so long time ago, performing anesthesia and single-lung ventilation were considered impossible in patients with spirometry values below $50 \%$ of normal. Following recent body of knowledge, this was just as simple as incorrect since in patients suffering from severe emphysema surgical procedures offer better survival and quality of life.

In this article, we give a very brief insight into modern anesthesia to allow thoracic surgery in severely ill patients with special respect to emphysema surgery.

\section{Preoperative considerations}

Especially patients with particularly impaired lung function, poor diffusing capacity and/or homogenous emphysema carry a high risk for mortality in emphysema surgery (3). For the anesthesiologist, the approach to preoperative evaluation is as individual as the often multimorbid patient. It must be guided by the aim to gather relevant information to optimize the medical condition and avoid complications; however, giving a cook-book recipe for preoperative evaluation is far from being reasonable. Surely, emphysema patients often suffer from relevant afflictions like coronary artery or arterial occlusive disease that can be camouflaged by emphysema related reduction in capacity. Most of the time, thoroughly evaluation of the medical history, the lung-function (X-ray, CT-scan, spirometry) and cardiac performance (right heart failure?) as well as any attempt to improve lung function are performed prior to or in context of the surgical evaluation. ECG and blood-testing to detect anemia, coagulopathy or organ dysfunction should be performed since some of the disorders (e.g., anemia) can be improved preoperatively. Moreover, an attentive evaluation must be taken to the upper airway to detect intubation problems; the ability of the patient to collaborate e.g., for placement of regional anesthesia or for awake procedures must be assessed.

Self-evident, the patient's consent based upon plain information and explanation of alternative ways in adapted language is necessary, especially when the patient's collaboration is mandatory.

The preparation for anesthesia and the line-up depends on the individual condition and institutional practice. Invasive monitoring (e.g., arterial line, central venous line, cardiac output monitoring, swan-ganz-catheter, processed EEG, blood-gas analysis) that exceed standard-monitoring following international guide-lines (ECG, pulse oximetry, blood pressure monitoring, carbon dioxide monitoring) should be tailored according to patient's needs. However, a good running venous line to tackle hemorrhage is one thing that should never be forgotten.

\section{State-of-the-art thoracic anesthesia and novel anesthesiological perspectives}

Today the most common, comfortable and most familiar way to perform anesthesia for thoracic surgery is a conventional general anesthesia using a double lumen tube (DLT) for airway management, combined with regional anesthesia and advanced monitoring like arterial blood pressure measurement.

It is considered comfortable and safe by most anesthesiologist because of the aim to have maximum control over parameters like artificial respiration with oxygenation, decarboxylation and pressure limitation for lung protective ventilation, analgesia and depth of anesthesia and hemodynamics.

Unfortunately this familiar package of anesthesiological care ignores an important topic, namely the goal to avoid baro-, volu-, atelect-, bio- and pressure-trauma that is almost inevitable linked to artificial ventilation. Particularly important for patients with severe lung emphysema, any kind of artificial ventilation can injure the remaining operating gas exchange surface of the lung. So, why not avoiding artificial ventilation or make artificial ventilation safe?

\section{Is there a possibility for spontaneous breathing during thoracic surgery?}

In the past few years the non-intubated video-assisted thoracoscopic surgery (NIVATS) has been intensively researched. It emerges as a rising alternative to the conventional intubated VATS under general anesthesia (GAVATS) (4,5). The successful implementation of the NIVATS technique is based on many parameters:

- Experienced and skilled team; 
- Well selected patients;

- Selected surgical procedures;

- Strict time management;

- Well-structured communication with the patient and within the team;

- Advanced and patent-tailored monitoring (e.g., endtidal capnometry);

- Well designed (analogo)sedation protocols;

- Patient-tailored regional anesthesia;

- Well prepared fallback levels in case of surgical or anesthesiological complications.

Because of all those considerations, the number of patients that qualify for NIVATS is rather small. This makes controlled trials evaluating NIVATS versus GAVATS rather challenging. However, many studies highlight several advantages of NIVATS such as reduction of postoperative complications, a shorter hospital stay and a decrease of perioperative mortality and morbidity; on the other hand, in times of focus on economics and standardized processes in the operating theatres, general anesthesia as mentioned in the introduction could be less time consuming in preparation and probably provides more comfort, for the patient and the treating physicians as well.

Is NIVATS thus favorable? We do not know on clear evidence basis, but for designated groups of patients it is safe and most likely advantageous.

\section{Can we optimize state-of-the-art thoracic anesthesia?}

Ancient approaches to sedate and ventilate fully lined up all patients after thoracic surgery on ICU for a couple of hours or days are surely outdated. The golden principle to do just enough to do a good job surely holds true in this context meaning that monitoring, choice of drugs and the perioperative resource utilization all have to be adapted to individual patient's needs.

General anesthesia necessitates modern short-acting medication, preferably Propofol, Remifentanil and Rocuronium to optimize timing of anesthesia and to make sure that the patient is fully awake at the end of surgery with all the connected benefits. Volatile anesthetics might have the disadvantage to influence the hypoxic vasoconstriction of the separated lung making SLV rather difficult. Moreover, keeping in mind that gas might leak aside a leaking endotracheal tube or bronchus resulting in a pollution of the operating theatre, it appears favorable to avoid volatiles. However, to date there is no sound evidence to completely abandon volatile anesthetics in thoracic surgery (6).

Usually, protective ventilation is applied to avoid high peak pressures and high inspiratory Oxygen fraction. The impact of tidal volumes appears to be of less magnitude (7). As well, fluid management should rather be restrictive to avoid overload.

EEG-monitoring (e.g., bispectral index BIS) can help to control timing and depth of anesthesia, namely to reduce negative effects of both oversedation and too shallow anesthesia (awareness, unintended movements or coughing during surgery). An arterial line is minimal invasive and allows to detect rapid changes in hemodynamics. In our view, central venous lines should carefully be considered; frequently their use does only offer a small comfort.

Relaxometry is obligatory when using muscle relaxants to assure both adequate relaxation according to surgical needs and to avoid a relaxation hangover. Nevertheless, the use of sugammadex should be handled generously to prevent postoperative respiratory failure by residual muscular relaxation.

Indeed, pain is a major topic in thoracic surgery (8). Regional techniques promise favorable effects. Epidural analgesia, paravertebral block, musculus serratus anterior block, intercostal block and the musculus erector spinae block have been widely studied. The thoracic epidural catheter is the most common and effective way to suppress acute pain and even to reduce the incidence of postthoracotomy-syndrome. On the other side there are a lot of contraindications and possible severe complications as well. Especially the multimorbid patients frequently need anticoagulation that precludes neuraxial blocks. Thus, many patients are not qualified for this effective method. A good compromise of risk and benefit can be the musculus erector spinae block/catheter; technically it belongs to the peripheral regional anesthesia respectively fascia block, so there are no contraindications referring to coagulopathy (9). It is easy to apply by using ultrasound guided canula control so it can even be applied in patients under general anesthesia. Thus it is even suitable for patients who are not able to take special posture such as sitting quietly for receiving an epidural catheter.

\section{Novel approaches}

Since sophisticated surgical techniques were introduced and validated in recent years, even patients suffering from an end-stage reduced pulmonary reserve can profit from surgery so that conventional anesthesiological approaches 
frequently reach their limits. SLV as the gold standard to allow lung-surgery unfortunately triggers a cascade of problems especially in patients with emphysema and bullae: The use of a DLT in comparison to a conventional tube necessitates higher-pressure levels due to the smaller diameter of each single lumen. The pressure can double when only one lung is ventilated (10). Positive pressure ventilation always means stress for the lung, especially via triggering an inflammatory reaction leading to a ventilator associated lung injury (VALI). Not to forget that in the non-ventilated lung inflammation can occur because of hypoxia and reoxygenation (11). Moreover, a single, largely compromised lung might not be sufficient to ensure pulmonary gas exchange during surgery. To minimize these problems different approaches have been developed and are successfully brought into clinical practice.

Avoiding intratracheal intubation: non-intubated thoracic surgery (NITS)

Turning away from the paradigm of general anesthesia and controlled SLV as a condition sine qua non for thoracic surgery, a variety of different surgical procedures have be widely performed in non-intubated, spontaneously breathing patients (12). A lot of reports have proven that these procedures can be performed safely and may even have advantages in different kinds of surgery like for example lung volume reduction (LVRS) (13).

Most procedures are performed in mild (analogo) sedation using different medications to improve patients' comfort and facilitate hemodynamic management. Devices for securing the airway are thus avoided; the use of a laryngeal mask, however, is also described. The patients should be supplemented with oxygen and, if necessary, intraoperative noninvasive ventilation (NIV) can be applied. Measurement of end-tidal carbon dioxide should be done for monitoring ventilation and is easily achieved by placing the probe line under an oxygen mask. A moderate degree of hypoxia and/or hypercapnia can be tolerated. Roughly, although there are no fixed limits validated in literature, a saturation above $90 \%$ and a $\mathrm{pH}$ in arterial blood above 7.2 are considered to be safe.

Analgosedation is mostly based on a patient-tailored dose of continuously infused Propofol and an opiate, often Remifentanil. Dexmedetomidine is also a good option as it is widely used in a variety of interventional procedures (13) up to high risk interventions like transapical aortic valve replacement (14) but also in NITS (15). Monitoring the depth of sedation can be facilitated by a processed EEG.

Analgesia is frequently achieved by thoracic epidural anesthesia (TEA) as gold standard, paravertebral or intercostal block or other regional anesthesia techniques. The goal is to reach an anaesthetized level between Th2 and Th10. To prevent coughing, a vagal blockade can be performed by the surgeon under thoracoscopic view to optimize conditions even for complicated procedures (16). Another possibility is the inhalation of aerosolized lidocaine to inhibit cough reflex $(11,15)$.

In case of persistent hypoxia, hypercapnia, intrathoracic bleeding or extreme patient discomfort conversion to general anesthesia might become necessary. Since the patient mostly is placed in lateral position, this can be challenging. Especially when the patient's gas exchange is decompensating, a rapid and pre-planned algorithm should be implemented. Different approaches are described, like temporary closing the chest, inserting a chest tube, turning the patient back into prone position and then inducing anesthesia and placing an endotracheal tube (17). But also intubating the patient in the lateral position by video laryngoscopy is considered a reasonable approach. The procedure is then either discontinued (if possible and reasonable) or finished in conventional SLV. In fact, conversion rate appears to be below ten percent $(11,16,18)$.

Contraindication for awake procedures are related to the surgery itself (demanding operation or emergency procedure, previous thoracic surgery), to the patient (refusal of the method or uncooperative patient, severe obesity, hemodynamic instability) or the regional anesthesia (impaired coagulation due medication or other reasons, allergy to local anesthetics, anatomical conditions).

\section{Avoiding the need of ventilation: extracorporeal membrane oxygenation}

There are patients suffering from extensively reduced pulmonary reserve that turn any anesthesia, mechanical ventilation and surgery into an unacceptable risk. However, for some of these patients' modern surgical procedures promise a markedly improved survival and quality of life. Lung volume reduction, tracheal surgery or tumor surgery in context of severely limited pulmonary function are possible scenario. In these cases, avoiding mechanical ventilation by extracorporeal devices (e.g., veno-venous Extracorporeal Membrane Oxygenation and decarboxylation, vv-ECMO) is an optional treatment. The principle of $\mathrm{vv}-\mathrm{ECMO}$ is to continuously drain venous blood via a large lumen canula, pump it through an artificial lung for oxygenation and decarboxylation and then reinfuse it into the venous system. Indeed, ECMO is an established treatment for patients with acute respiratory 
failure like ARDS; there becomes more and more literature available about its intraoperative use to allow or facilitate high-risk surgery (19-21).

Depending on the goal of treatment there are of course differences in the technique of cannulation and anticoagulation. In case hypercapnia is the primary problem low flow $(1-1.5 \mathrm{~L} / \mathrm{min}) \mathrm{vv}-\mathrm{ECMO}$ with a single twin port double lumen canula in the upper jugular vein $(22 \mathrm{~F})$ or femoral vein $(24 \mathrm{~F})$ can be applied.. If there is also the need of oxygenation support, higher flow rates are needed and two cannulas, normally inserted in the jugular (17-19 F) and femoral vain (19-23 F) are necessary. In this case, ultraprotective ventilation with low tidal volumes during the surgical intervention or even a complete stop of pulmonary ventilation are possible.

To prevent thromboembolic complications anticoagulation is needed; an instant that does not create much enthusiasm in the surgeon's mind since it might increase the risk for bleeding complications. There are several suggestions for dosing Heparin to reach a target PTT of 1.5-2.5 of the norm or even lower (22); even waiving any anticoagulation might be reasonable since the ECMO-circuits are heparin coated. This might be enough to allow short time runs of $\mathrm{vv}$-ECMO during surgery. However, any underlying, unequivocal data is focuses on long time support on the ICU and transferring the statements to the operation theatre requires caution. In our view, for the short intraoperative use and when modern circuits are employed, the admission of a single heparin bolus $(2,000-4,000$ I.E.) appears to be enough to safely do the job (18). Mind that extracorporeal circulation, among others, triggers inflammation and influences thrombocyte function so that coagulation per se is likely to be affected.

When surgery is finished, the vv-ECMO can most of the times be removed in the operation theater or, if necessary, the patient can be weaned from it postoperatively on ICU within hours or a few days.

For the future selected groups of patients may benefit from the combination of extracorporeal pulmonary support and NITS but putting this issue on an evidence-based level will require much more research in this field (e.g., anticoagulation and regional anesthesia).

\section{Acknowledgments}

Funding: None.

\section{Footnote}

Provenance and Peer Review: This article was commissioned by the Guest Editor (Bassam Redwan) for the series "Lung Emphysema" published in Annals of Translational Medicine. The article was sent for external peer review organized by the Guest Editor and the editorial office.

Conflicts of Interest: All authors have completed the ICMJE uniform disclosure form (available at http:// dx.doi.org/10.21037/atm-2019-le-06). The series "Lung Emphysema" was commissioned by the editorial office without any funding or sponsorship. The authors have no other conflicts of interest to declare.

Ethical Statement: The authors are accountable for all aspects of the work in ensuring that questions related to the accuracy or integrity of any part of the work are appropriately investigated and resolved.

Open Access Statement: This is an Open Access article distributed in accordance with the Creative Commons Attribution-NonCommercial-NoDerivs 4.0 International License (CC BY-NC-ND 4.0), which permits the noncommercial replication and distribution of the article with the strict proviso that no changes or edits are made and the original work is properly cited (including links to both the formal publication through the relevant DOI and the license). See: https://creativecommons.org/licenses/by-nc-nd/4.0/.

\section{References}

1. Brodsky JB, Lemmens HJM. The history of anesthesia for thoracic surgery. Minerva Anestesiol 2007;73:513-24.

2. Li Q, Zhang X, Xu M, et al. A retrospective analysis of 62,571 cases of perioperative adverse events in thoracic surgery at a tertiary care teaching hospital in a developing country. J Cardiothorac Surg 2019;14:98.

3. van Agteren JE, Carson KV, Tiong LU, et al. Lung volume reduction surgery for diffuse emphysema. Cochrane Database Syst Rev 2016;10:CD001001.

4. Beck-Schimmer B, Bonvini JM, Braun J, et al. Which Anesthesia Regimen Is Best to Reduce Morbidity and Mortality in Lung Surgery?: A Multicenter Randomized Controlled Trial. Anesthesiology 2016;125:313-21.

5. Zhang K, Chen HG, Wu WB, et al. Non-intubated videoassisted thoracoscopic surgery vs. intubated video-assisted 
thoracoscopic surgery for thoracic disease: a systematic review and meta-analysis of 1,684 cases. J Thorac Dis 2019;11:3556-68.

6. Sunaga H, Blasberg JD, Heerdt PM. Anesthesia for nonintubated video-assisted thoracic surgery. Curr Opin Anaesthesiol 2017;30:1-6.

7. Amar D, Zhang H, Pedoto A, et al. Protective Lung Ventilation and Morbidity After Pulmonary Resection: A Propensity Score-Matched Analysis. Anesth Analg 2017;125:190-9.

8. Elmore B, Nguyen V, Blank R, et al. Pain Management Following Thoracic Surgery. Thorac Surg Clin 2015;25:393-409.

9. De Cassai A, Bonvicini D, Correale C, et al. Erector spinae plane block: a systematic qualitative review. Minerva Anestesiol 2019;85:308-19

10. Spaeth J, Hojnik A, Ott M, et al. Characteristics of Double-Lumen Tubes Determine Bronchial Airway Pressure. J Cardiothorac Vasc Anesth 2016;30:954-60.

11. Mineo TC, Tacconi F. From "awake" to "monitored anesthesia care" thoracic surgery: A 15 year evolution. Thorac Cancer 2014;5:1-13.

12. Akopov A, Egorov V, Deynega I, et al. Awake videoassisted thoracic surgery in acute infectious pulmonary destruction. Ann Transl Med 2015;3:100.

13. Pompeo E, Rogliani P, Palombi L, et al. The complex care of severe emphysema: role of awake lung volume reduction surgery. Ann Transl Med 2015;3:108.

14. Barends CRM, Absalom A, van Minnen B, et al. Dexmedetomidine versus Midazolam in Procedural Sedation. A Systematic Review of Efficacy and Safety.

Cite this article as: Woldt P, Kruse P, Ellger B. Anesthesiological considerations in emphysema surgery. Ann Transl Med 2020;8(21):1470. doi: 10.21037/atm-2019-le-06
PLoS One 2017;12:e0169525.

15. Peng G, Liu M, Luo Q, Chen H, Yin W, Wang W, et al. Spontaneous ventilation anesthesia combined with uniportal and tubeless thoracoscopic lung biopsy in selected patients with interstitial lung diseases. J Thorac Dis 2017;9:4494-501.

16. Park HS, Kim KM, Joung KW, et al. Monitored anesthesia care with dexmedetomidine in transfemoral percutaneous trans-catheter aortic valve implantation: two cases report. Korean J Anesthesiol 2014;66:317-21.

17. Guarracino F, Gemignani R, Pratesi G, et al. Awake palliative thoracic surgery in a high-risk patient: one-lung, non-invasive ventilation combined with epidural blockade. Anaesthesia 2008;63:761-3.

18. Chen KC, Cheng YJ, Hung MH, et al. Nonintubated thoracoscopic surgery using regional anesthesia and vagal block and targeted sedation. J Thorac Dis 2014;6:31-6.

19. Redwan B, Semik M, Dickgreber N, et al. Single site cannulation veno-venous extracorporeal lung support during pulmonary resection in patients with severely compromised pulmonary function. ASAIO J 2015;61:366-9.

20. Redwan B, Ziegeler S, Freermann S, et al. Intraoperative veno-venous extracorporeal lung support in thoracic surgery: a single-centre experience. Interact Cardiovasc Thorac Surg 2015;21:766-72.

21. Rehers S, Beiderlinden M, Ziegeler S. Extrakorporale Membranoxygenierung in der Thoraxchirurgie: die Sicht des Anästhesisten. Zentralbl Chir 2019;144:86-92.

22. Brogan TV, Lequier L, Lorusso R. Extracorporeal life support: The ELSO Red Book. 5th edition, 2017. 